\title{
Correlation between degree of subvoxel spinal cord compression measured with super-resolution tract density imaging and neurological impairment in cervical spondylotic myelopathy
}

\author{
Benjamin M. Ellingson, PhD, ${ }^{1-3}$ Noriko Salamon, MD, PhD, ${ }^{1}$ Davis C. Woodworth, BS, \\ and Langston T. Holly, MD ${ }^{4}$
}

Departments of ${ }^{1}$ Radiological Sciences, ${ }^{2}$ Biomedical Physics, ${ }^{3}$ Bioengineering, and ${ }^{4}$ Neurosurgery and Orthopaedics, David Geffen School of Medicine, University of California, Los Angeles, California

\begin{abstract}
OBJECT The purpose of this study was to explore the use of super-resolution tract density images derived from probabilistic diffusion tensor imaging (DTI) tractography of the spinal cord as an imaging surrogate for microstructural integrity and functional impairment in patients with cervical spondylosis.

METHODS Structural MRI and DTI images were collected for 27 patients with cervical spondylosis with $(n=21)$ and without $(n=6)$ functional impairment as defined by the modified Japanese Orthopaedic Association Scale (mJOA). DTI was performed axially through the site of compression in a total of 20 directions with 10 averages. Probabilistic tractography was performed at $0.5-\mathrm{mm}$ isotropic spatial resolution using the streamline technique combined with constrained spherical deconvolution. The following measurements were calculated for each patient: maximum tract density at the site of compression, average tract density in rostral normal-appearing spinal cord, and the ratio of maximum density to normal density.
\end{abstract}

RESULTS Compared with normal tissue, the site of compression exhibited elevated fiber tract density in all patients, and a higher fiber tract density was also noted in focal areas at the site of compression in patients with functional impairment. There was a strong negative correlation between maximum tract density and mJOA score $\left(R^{2}=0.6324, p<\right.$ $0.0001)$ and the ratio of maximum tract density to normal tract density $\left(R^{2}=0.6647, p<0.0001\right)$. When grouped according to severity of neurological impairment (asymptomatic, mJOA score of 18; mild, mJOA score of 15-17; moderate, mJOA score of 11-14; and severe, mJOA score < 11), the results showed a significant difference in the ratio between severe and both no impairment $(p=0.0009)$ and any impairment $(p=0.036)$. A ratio of maximum fiber tract density at the site of compression to fiber tract density at C-2 greater than 1.45 had $82 \%$ sensitivity and $70 \%$ specificity for identifying patients with moderate to severe impairment (ROC AUC $=0.8882, p=0.0009)$.

CONCLUSIONS These results support the use of DTI as a surrogate for determining spinal cord integrity in patients with cervical spondylosis. Probabilistic tractography provides spinal cord microstructural information that can help discern clinical status in cervical spondylosis patients with varying degrees of neurological impairment.

http://thejns.org/doi/abs/10.3171/2014.10.SPINE14222

KEY WORDS diffusion tensor imaging; DTI; spinal cord; cervical spondylotic myelopathy; biomarker; stenosis; modified Japanese Orthopaedic Association Scale; tractography

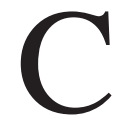
ERVICAL spondylosis is a chronic degenerative condition of the spine ${ }^{3}$ that is often considered part of a spectrum, and it can progress from an asymptomatic state to varying degrees of radiculopathy and/or myelopathy. Cervical spondylosis occurs in as many as $95 \%$ of men and $70 \%$ of women between 60 and 65 years old, ${ }^{15,42}$ and cervical myelopathy is the most common cause of acquired spinal cord dysfunction in the middle aged and elderly. ${ }^{41}$

Advanced cervical spondylosis, as manifested by degeneration of the osseous and soft-tissue structures, can potentially result in myelopathy due to spinal cord com-

ABBREVIATIONS AUC = area under the ROC curve; $C S M=$ cervical spondylotic myelopathy; $D T I=$ diffusion tensor imaging; $\mathrm{mJOA}=$ modified Japanese Orthopaedic Association Scale; ROC = receiver-operator characteristic; SEA = snake-eye appearance; TDI = tract density imaging.

SUBMITTED March 3, 2014. ACCEPTED October 14, 2014.

INCLUDE WHEN CITING Published online March 6, 2015; DOI: 10.3171/2014.10.SPINE14222.

DISCLOSURE Funding was received through the following NIH/NINDS grants: no. 1R21NS065419-01A1 (to L.T.H. and N.S.) and no. 1R01NS078494-01A1 (to L.T.H., N.S., and B.M.E.). 
pression. It is not uncommon for surgery to be advocated for in these patients, even when they are completely asymptomatic or only mildly symptomatic. As such, it is of great importance to develop an imaging surrogate that can be used to better analyze areas of compression, estimate neurological impairment, and quantify the extent of spinal cord damage to allow us to more optimally identify patients who actually need surgical intervention.

MRI is the gold standard for noninvasive assessment of the spinal cord; however, standard MRI measures of spinal cord damage including degree of compression ${ }^{29,34,42}$ or MRI signal change ${ }^{13,19-23,26,30}$ have not demonstrated a robust correlation with neurological function. Diffusion tensor imaging (DTI) is an MRI technique that is sensitive to the magnitude and orientation of water self-diffusion, providing unique insight into soft-tissue microstructure. ${ }^{33}$ DTI has been shown to be superior to routine MRI for identifying lesions in the spinal cord, $9,11,12$ and numerous studies have suggested this technique may have diagnostic utility. 4,9,12,16,19,32,36,39

The directional dependence of nerve fibers in the brain and spinal cord results in diffusion anisotropy, which can be quantified using DTI. Additionally, this diffusion anisotropy can be used to model neural tract orientation, or "pseudo-axonal tracts," using DTI fiber tractography techniques. In the current study we performed probabilistic tractography in patients with cervical spondylosis with or without neurological impairment and generated superresolution tract density imaging $(\mathrm{TDI})^{5-7}$ at high spatial resolution based on these simulations. We then used quantitative measurements from TDI to estimate spinal cord integrity and neurological impairment in patients with varying degrees of cervical spondylosis. We hypothesized that patients with high fiber tract density at the site of compression would have greater neurological deficits than those patients with lower measures of tract density, suggesting a higher degree of microstructural compression of spinal tissue results in greater functional impairment.

\section{Methods}

\section{Patients}

A prospective study was carried out to describe quantitative TDI images of the cervical spinal cord in 27 cervical spondylosis patients (14 females and 13 males; age range $21-75$ years; mean age 62 years) with $(n=21)$ or without $(n$ $=6$ ) neurological impairment. The patients without neurological symptoms had primary complaints of neck pain. All patients had at least mild to moderate spinal canal stenosis evidenced on standard MRI. The modified Japanese Orthopaedic Association (mJOA) ${ }^{40}$ scale was used as the functional assessment measure. The patients' mean mJOA score $( \pm \mathrm{SD})$ was $14.9 \pm 2.9$ (range $8-18)$. All procedures complied with the principles of the Declaration of Helsinki and were approved by the institutional review board at our institution.

\section{Clinical Presentation}

Gait difficulty, the most common presenting complaint, was noted in 11 patients; difficulty using their hands was documented in 10 patients; a history of neck pain was noted in 9; and paresthesias in the upper extremities was shown in 6.

\section{Standard MRI Sequences and Evaluation}

Imaging procedures consisted of both routine clinical MRI scans and DTI scans acquired in a 3.0-T MRI scanner (3T TrioTim; Siemens Healthcare) using a standard spine coil array for radiofrequency reception. Routine clinical MRI consisted of T1-weighted and T2-weighted sequences in the sagittal plane and T2-weighted images in the axial plane. The presence of spinal cord T2 hyperintensity, so-called snake-eye appearance (SEA) of T2 hyperintensity, 1,25,31,37 anterior-posterior diameter of the cord at the level of highest compression, and the Torg-Pavlov ratio $^{29}$ (ratio of anterior-posterior diameter to thickness of the vertebral body) was documented and used for subsequent comparisons with TDI metrics.

\section{Diffusion Tensor Imaging}

Axial diffusion-weighted images were acquired through the level of most significant canal narrowing. Excitation consisted of a custom 2D, spatially selective radiofrequency excitation pulse and a reduced field of view echo planar imaging readout with ramp sampling (Zoomed-EPI). The TE/TR was set to $73 \mathrm{msec} / 3000 \mathrm{msec}$; matrix size was 48 $\times 128$; FOV, $53 \times 140 \mathrm{~mm}$; slice thickness, $4 \mathrm{~mm}$ with no gap; number of averages, 10; and there were 20 diffusion sensitizing directions with $b=500 \mathrm{sec} / \mathrm{mm}^{2}$ and a single $b$ $=0 \mathrm{sec} / \mathrm{mm}^{2}$ image. After acquisition of diffusion-weighted images, eddy-current and motion correction were performed using a 12-degree of freedom affine transformation using FSL (FMRIB; Oxford, UK; http://www.fmrib. ox.ac.uk/fsl/).

\section{Fiber Tracking}

Fiber tracking was performed using the MRtrix software package (Brain Research Institute, http://www.brain. org.au/software/), based on the probabilistic streamlines method $^{2,28}$ combined with the constrained spherical deconvolution technique ${ }^{35}$ to model multiple fiber orientations. Tracking was performed by randomly seeding throughout the cervical spinal cord (1 million seeds placed throughout a $25-\mathrm{cm}^{3}$ volume of the cervical cord, or 40,000 seeds/ $\mathrm{cm}^{3}$ ) using a $0.1-\mathrm{mm}$ step-size, a maximum angle between steps of $20^{\circ}$, and a maximum harmonic order of $I_{\max }=4$.

\section{Tract Density Imaging}

To generate quantitative maps of fiber tract density, fiber tracking was first performed using whole-spine seeding (e.g., 1,000,000 seeds throughout the cervical cord), including areas of $\mathrm{T} 2$ hyperintensity that might be due to edema, inflammation, or myelomalacia. From the resulting fiber tracts, the total number of tracts present in each element on a high-resolution grid of $0.5-\mathrm{mm}$ isotropic resolution were calculated. These TDI images use the continuity information contained in the streamlines to quantify subvoxel tract density. The resulting quantitative TDI images were used for subsequent analysis. 


\section{Regions of Interest}

Manual segmentation of the spinal cord was performed for the whole cord (no gray/white matter distinction) at each axial image slice location, using the T2-weighted anatomical images. TDI measurements at the site of highest compression, including voxels in areas of T2 hyperintensity, were used for statistical comparisons.

\section{Statistical Analysis}

Linear regression was used to test whether the maximum fiber tract density at the site of compression (normalappearing tissue) and the ratio of maximum fiber tract density to average density at C-2 correlated with the mJOA score. Additionally, a Kruskal-Wallis test and Dunn's test for multiple comparisons were used to identify differences in TDI measurements between patients with no impairment (mJOA score of 18), mild impairment (mJOA score of 15-17), moderate impairment (mJOA score of 11-14), and severe impairment (mJOA score of < 11). Lastly, receiver-operator characteristic (ROC) analysis was used to determine the sensitivity and specificity for TDI measurements to differentiate stenosis patients with at least mild impairment (mJOA score < 18) from stenosis patients without myelopathy (mJOA score of 18), as well as patients with at least moderate impairment (mJOA score of $<15$ ) from those with mild or no impairment (mJOA score of $\geq 15$ ). The area under the ROC curve (AUC) was used as a measure of TDI performance. Mean values are presented \pm SD.

\section{Results}

\section{Standard MRI}

There was no significant correlation between mJOA score and the diameter of the spinal canal (Fig. 1A; $\mathrm{R}^{2}$ $=0.0419, \mathrm{p}=0.3055)$. Similarly, no significant correlation was observed between mJOA and the Torg-Pavlov ratio (Fig. 1B; $\mathrm{R}^{2}=0.1004, \mathrm{p}=0.1073$ ); however, the ratio trended toward lower values for increasing neurological impairment. The presence of $\mathrm{T} 2$ signal changes within the cord was associated with lower mJOA score (Fig. 1C; ttest, $\mathrm{p}=0.0058)$. Of the 10 patients with $\mathrm{T} 2$ signal changes within the cord, 7 patients had a characteristic SEA lesion pattern. These 7 patients tended to have a lower mJOA score (mean mJOA score 11.6) than patients whose images showed T2 hyperintensity but no SEA pattern $(n=3$; mean mJOA score 16.3), but no considerable differences in Torg-Pavlov ratio or TDI metrics were observed.

\section{Tractography}

Compared with normal tissue at the $\mathrm{C}-2$ level, the site of compression exhibited elevated fiber tract density in all patients. In patients without neurological symptoms (mJOA score of $18 ; n=6$ ), subtle elevation in tract density compared with $\mathrm{C}-2$ was observed (Fig. 2A). In patients with mild impairment (mJOA score of $15-17 ; \mathrm{n}=11$ ), a focal area of increased tract density was evident that did not normally extend more than one spinal level (Fig. 2B). Patients with moderate impairment (mJOA score of 11-14; $n=7$ ) had elevated tract density at the area of compression
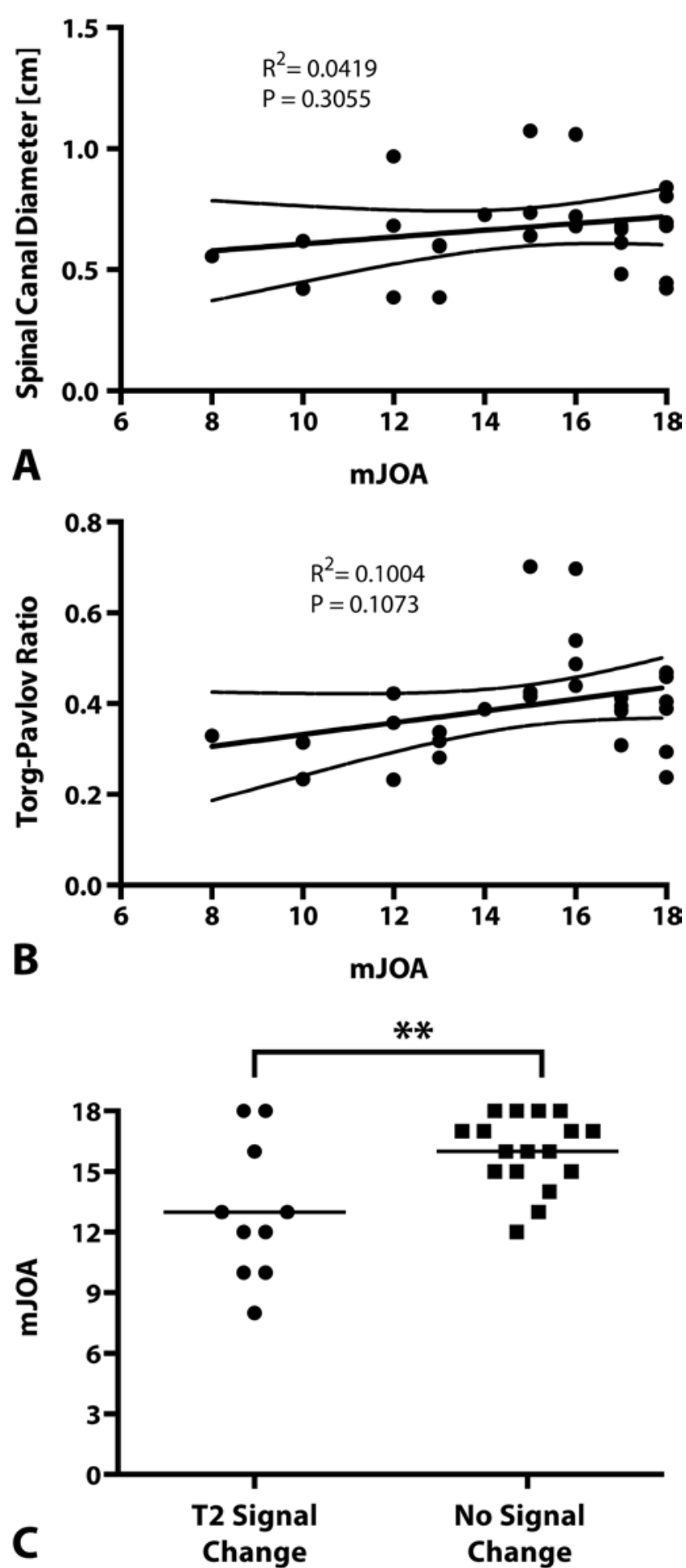

FIG. 1. Association between anatomical MRI measurements and neurological impairment. A: Linear correlation between anterior-posterior spinal canal diameter at the site of stenosis and $\mathrm{mJOA}\left(R^{2}=0.0419, p=\right.$ 0.3055). B: Linear correlation between the Torg-Pavlov ratio, or ratio of spinal canal diameter to vertebral body diameter, and $\mathrm{mJOA}\left(\mathrm{R}^{2}=0.1004\right.$, $p=0.1073)$. C: Comparison of mJOA scores between patients with intramedullary T2 signal changes $(n=10)$ and those without T2 signal change $(n=17)$, suggesting patients with T2 signal changes have a lower mean mJOA score (t-test, $p=0.0058$ ). Circles indicate patients with intramedullary $\mathrm{T} 2$ change. Squares represent patients without intramedullary $\mathrm{T} 2$ change. Horizontal lines indicate the group mean. ${ }^{* *} p<0.01$. 

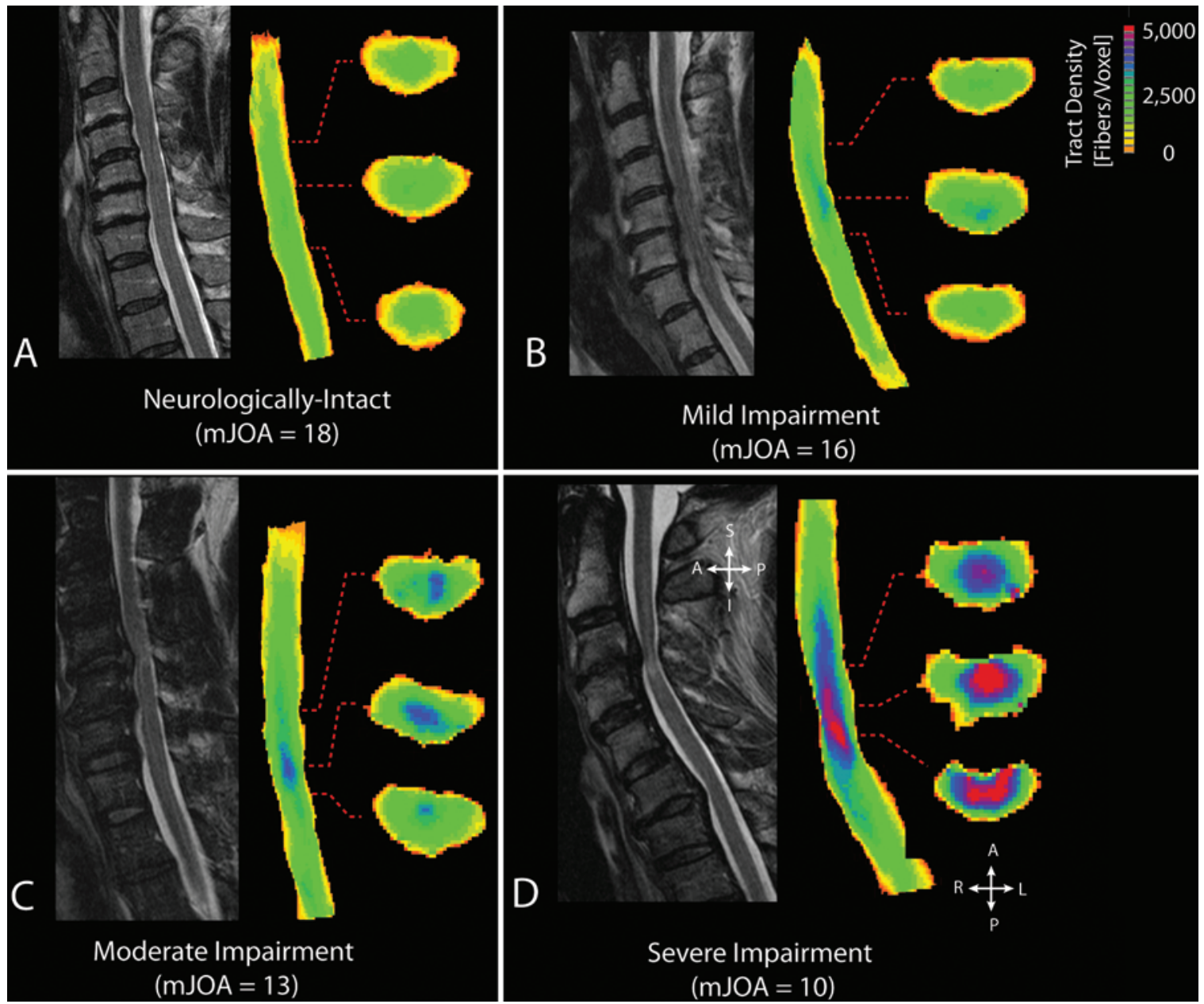

FIG. 2. Sagittal T2-weighted MR images and super-resolution TDI color maps of the spinal cord in cervical spondylosis patients with and without neurological impairment. A: A 58-year-old female patient with no neurological impairment (mJOA score of 18) and very little spinal cord compression. TDI shows little to no change in fiber tract density along the length of the spinal cord. B: A 71-year-old female patient with mild neurological impairment (mJOA score of 16) and stenosis at C4-5 and C6-7. TDI shows a localized area of high fiber density in the dorsal columns at the site of greatest stenosis. C: A 53-year-old male patient with moderate neurological impairment (mJOA score of 13 ) and stenosis at C4-5 and C5-6, with a T2 signal change on anatomical MRI. TDI shows a focal area of high fiber density in the central areas of the spinal cord at the level of greatest stenosis, and areas of relatively high fiber density extending rostral and caudal to adjacent levels. D: A 57-year-old female patient with severe neurological impairment (mJOA score of 10) and stenosis at C3-4, with T2 signal changes on anatomical MRI. TDI shows a focal area of extremely high fiber density and compressed fibers extending more than 2 levels adjacent to the site of stenosis. Note that regions of red/fuchsia within the center of the cord in D represent a region of high fiber density. Figure is available in color online only.

beyond that of patients with mild symptoms and appeared to have regions exhibiting increased tract density that extended across more than one spinal level (Fig. 2C). In patients with severe impairment (mJOA score of $<11$; $\mathrm{n}=$ 3 ), we observed focal areas of very high fiber tract density that extended more than two spinal levels (Fig. 2D).

Consistent with these qualitative observations we observed a significant negative correlation between maximum fiber tract density at the site of compression and mJOA (Fig. 3A; $\mathrm{R}^{2}=0.6324, \mathrm{p}<0.0001$ ), suggesting that patients with a higher degree of functional impairment had a higher focal fiber tract density than patients with less neurological impairment. The sensitivity of maximum fiber tract density to mJOA score indicated a mean increase of approximately $198 \pm 30$ fibers per voxel for a single decrease in mJOA score. This measurement reflects the local fiber tract density, which is dependent on both the total number of fibers passing through the lesion and the cross-sectional area of the cord at the site of compression. The average fiber tract density in normal-appearing spinal tissue at the $\mathrm{C} 2$ level was $2575 \pm 82$ fibers/voxel for all patients. The ratio of maximum fiber tract density at the level of compression to average fiber tract density at C-2 significantly correlated with the mJOA score (Fig. 3B; $\mathrm{R}^{2}$ $=0.6647, \mathrm{p}<0.0001$ ). Additionally, the ratio of maximum fiber tract density at the level of compression to the average density at C-2 was significantly higher in patients exhibiting intramedullary T2 signal changes compared with patients showing no T2 signal changes within the cord (average ratio $1.62 \pm 0.1$ vs $1.39 \pm 0.05$; $\mathrm{t}$-test, $\mathrm{p}=0.045$ ), while the maximum fiber tract density measurement at the site of compression alone exhibited similar trends (aver- 

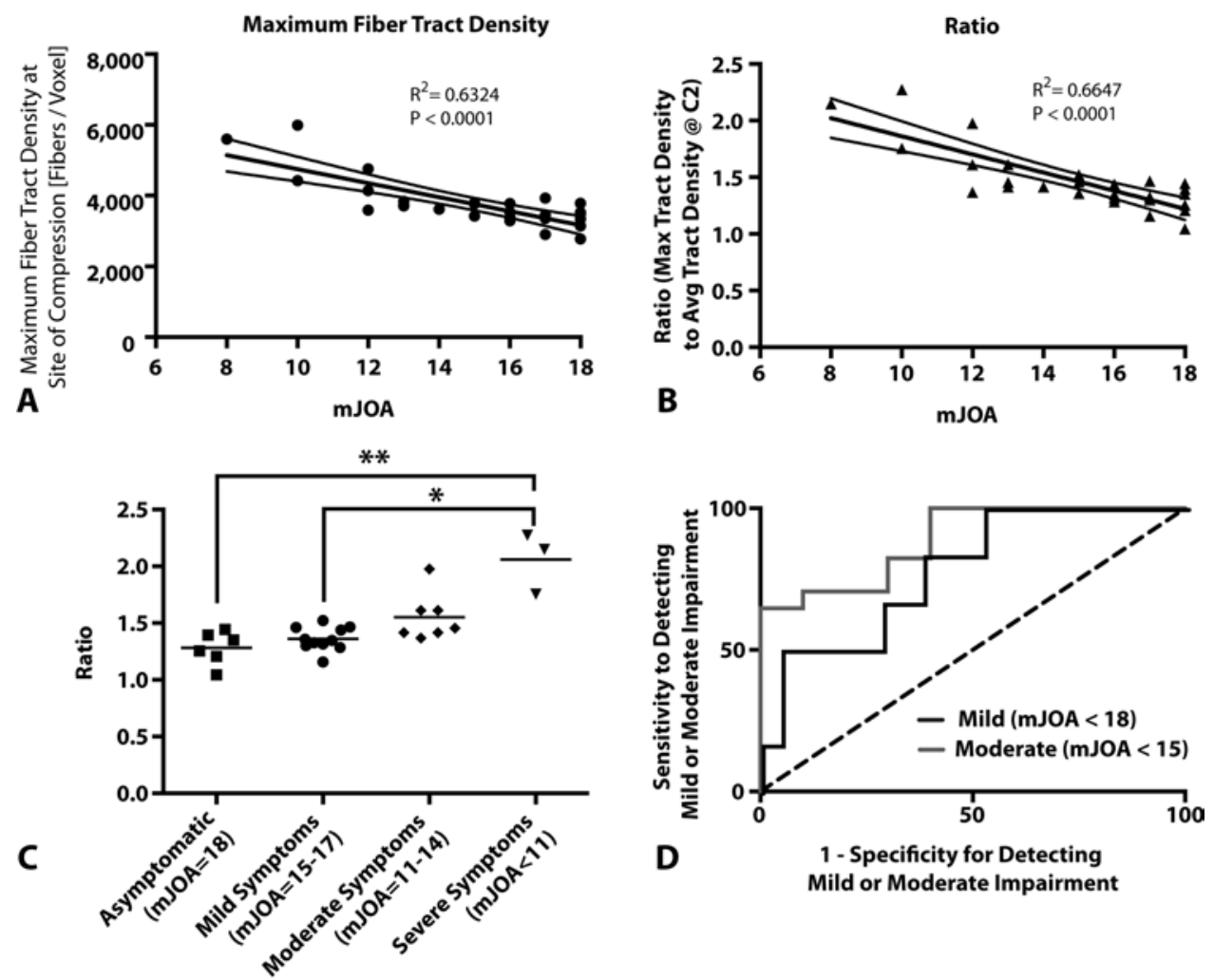

FIG. 3. Quantitative TDI measurements in cervical spondylosis. A: Linear correlation between measurements of the maximum fiber tract density at the site of stenosis and $\mathrm{mJOA}\left(R^{2}=0.6324 ; p<0.0001\right)$. B: Linear correlation between the ratio of maximum fiber tract density at the site of compression to normal tract density measured at $C-2$ and $m J O A\left(R^{2}=0.6647 ; p<0.0001\right)$. C: The ratio of maximum fiber tract density at the site of compression to normal tract density measured at C-2 for patients with mild, moderate, severe, and no neurological impairment showed significant differences between patients with severe and with mild symptoms or no symptoms. D: ROC characteristics for the ratio of maximum tract density at the site of compression to normal tract density.

age maximum density $4145 \pm 285$ vs $3601 \pm 120$; t-test, $p$ $=0.053)$.

When categorized by degree of impairment (asymptomatic, mild, moderate, severe, or no impairment), there was a significant difference in the ratio of maximum fiber tract density to normal tissue at C-2 and the degree of impairment (Fig. 3C; Kruskal-Wallis, $p=0.0039$ ). In particular, we observed a significant difference between fiber tract density in severely impaired patients and both patients with no or mild impairment (Dunn's test, $\mathrm{p}<0.05$ ). ROC analysis focusing on the ability for the ratio of maximum fiber tract density to normal density at C-2 to predict impairment suggested the ratio was able to significantly discern symptomatic patients (i.e., those with a mJOA score of $<18$ ) from asymptomatic patients (i.e., those with a mJOA score of 18) (Fig. 3D; ROC AUC = 0.7857, p = 0.036). In particular, a maximum fiber tract ratio at the site of compression greater than 1.4 had $83 \%$ sensitivity and $62 \%$ specificity for identifying patients with neurological deficits (positive predictive value $68.6 \%$; negative predictive value $78.5 \%$ ). Additionally, a ratio of maximum fiber tract density at the site of compression to fiber tract density at C-2 greater than 1.45 had $82 \%$ sensitivity and $70 \%$ specificity (positive predictive value $73.2 \%$; negative predictive value $79.6 \%$ ) for identifying patients with moderate to severe impairment (Fig. 3D; ROC AUC = 0.8882, p = 0.0009).

\section{Discussion}

Cervical spondylosis is ubiquitous, as it is encountered in the vast majority of middle-aged and older adults. In a subset of patients, advanced cervical spine degeneration can lead to significant neurological problems such as cervical spondylotic myelopathy (CSM). As the middleaged and older population continues to grow, one of the challenges will be the discernment and treatment of patients with similar degrees of cervical stenosis who have quite different neurological status. There is significant controversy in our field regarding whether asymptomatic or mildly affected patients with spinal cord compression related to advanced spondylosis should undergo surgery. One reason for this lack of consensus is the fact that traditional imaging modalities such as standard MRI provide relatively little quantitative information regarding the magnitude of spinal cord injury.

Despite the lack of quantification, the detection of traditional MRI changes has been shown to be advantageous for identifying severe changes within the cord. For example, intramedullary signal changes have been shown to be associated with a worse prognosis. In particular, the appearance of "snake eye" T2 hyperintense regions within the cord has been shown to be associated with a worse prognosis after decompressive surgery in patients with spinal cord compression. ${ }^{25}$ Histopathological studies have 
suggested this pattern of signal changes within the spinal cord may be due to central gray matter damage within the ventrolateral posterior column, ${ }^{24,25}$ a common site of compression. Consistent with these studies, we observed this lesion pattern in 7 of 10 patients with intramedullary signal changes, and patients with this type of pattern tended to have a lower mJOA score.

\section{DTI and Deterministic Versus Probabilistic Tractography}

In contrast to standard MRI, DTI has been shown to be highly sensitive in the quantification of spinal cord injury. ${ }^{9,11,12}$ The majority of previously published DTI studies in patients with cervical spondylosis have focused on utilizing DTI indices to predict functional impairment, noting that DTI measures of fractional anisotropy and mean diffusivity change during chronic compression of the spinal cord and may be related to axonal reorganization, degeneration, and/or atrophy. In particular, studies have shown that there is often an increase in mean diffusivity and decrease in fractional anisotropy at the site of chronic compression in patients with chronic spondylosis relative to neurologically intact control subjects, and these changes in diffusivity are proportional to the degree of neurological impairment. ${ }^{14,15,17}$ Although diffusion tractography is slightly dependent on the degree of diffusion anisotropy to estimate the primary direction of preferential diffusivity, the current study focused solely on quantification of parameters related to diffusion tractography and not DTI scalar indices.

Various studies have shown that tractography of the spinal cord can be useful for visualizing areas of compression and potentially predicting neurological impairment. ${ }^{4,8,12,18,27,38,39}$ Standard approaches to DTI tractography often involve deterministic algorithms for identifying path orientations, providing only a single set of fiber tracts for a given DTI data set. ${ }^{10}$ Deterministic algorithms, however, have inherent limitations such as the choice of initialization or seed placement, high sensitivity to estimated principal eigenvector orientation from the diffusion tensor, lack of straightforward methodology for identifying tract statistics, and the inability to deal with complex neural architecture including crossing fibers. To overcome these limitations, probabilistic DTI tractography algorithms were developed to estimate the probability of fiber trajectories given hundreds to thousands of simulated initial fiber trajectories, allowing for modeling of complex architecture including multiple fiber crossings, reduced error associated with noise, and the ability to quantify tract statistics. ${ }^{10}$ In the current study, we performed probabilistic tractography of the cervical spinal cord in patients with cervical spondylosis to visualize and quantify microscopic compression of neural tissue.

\section{Present Study}

Our results support the hypothesis that subvoxel compression of the human spinal cord due to cervical spondylosis can be detected, visualized, and quantified using super-resolution tract density images derived from probabilistic DTI tractography. In particular, we observed a strong negative correlation between fiber tract density and neurological impairment, suggesting that patients with larger functional deficits have a higher degree of compression than patients with fewer or less severe deficits. In contrast, standard MRI macroanatomical measurements, such as the spinal canal diameter or the Torg-Pavlov ratio, were not associated with the degree of neurological impairment. Although there is controversy in the management of mildly affected CSM patients, the medical literature is clear that patients with moderate and severe CSM are most optimally treated with surgical management. The results from our study indicate that tractography can identify patients with moderate and severe CSM with a high sensitivity, in contrast to standard MRI parameters. Thus, this microstructural spinal cord information can potentially be used to assist in the evaluation of patients with CSM.

To our knowledge, the finding that a significantly higher focal fiber tract density at the site of compression in patients with more severe neurological impairment has not been reported in the literature; however, a few studies have used fiber tractography of the spinal cord to provide insight into functional impairment in patients with cervical spondylosis. An early study by Facon et al. ${ }^{12}$ used deterministic tractography to visualize the site of compression and noted condensed fiber tracts, but they did not quantify these observations. A study by Chang et al. ${ }^{8}$ used deterministic tractography to examine 10 patients with cervical spondylosis and reported that a smaller proportion of tracts seeded rostrally from the site of compression were able to penetrate the site of compression; however, they did not note the presence of a focal increase in fiber density at the level of compression. Budzik et al. ${ }^{4}$ explored diffusion characteristics along fiber tracts obtained using deterministic tractography in 20 patients with cervical spondylosis, noting that diffusion characteristics in fiber tracts passing through the lesion were significantly different between symptomatic and asymptomatic patients. They did not discuss measurement of fiber tract density at the site of compression. Additionally, Wen et al..$^{38}$ used deterministic tractography in 15 healthy controls and 7 patients with cervical spondylosis and noted a decrease in the total number of fibers in the spinal cord in the patients with cervical spondylosis, but again they did not comment on fiber density at the site of highest compression in these cases.

On first glance our results may appear counterintuitive since we observed a localized increase in fiber tract density for an increasing neurological impairment, and an increased neurological impairment is typically thought to result from destruction or degeneration of spinal tracts. However, it is important to note that neurological impairment and T2 signal hyperintensity within the cord observed on imaging can be reversible after surgical intervention and that imaging patterns consistent with myleomalacia may not actually reflect true histopathological myleomalacia. For example, a study by Ramanauskas et al. ${ }^{31} \mathrm{dem}-$ onstrated that acute patterns of myelomalacia could be reversible and sometimes resolved spontaneously over time, whereas chronic patterns of myelomalacia often resulted in central cystic degeneration, syrinx formation, and atrophy of the cord, consistent with histological features of myelomalacia. Thus, it is conceivable that the majority of the patients in the current study did not exhibit chronic degeneration within the spinal cord consistent with true 
myelomalacia, since they did not have severe neurological impairment and we did not observe a localized decrease in fiber tract density with increasing neurological impairment. In fact, we observed a higher tract density at the site of compression in patients exhibiting intramedullary T2 signal changes when directly compared with those showing no T2 hyperintensity in the cord, suggesting that T2 signal changes within the cord were likely due to either inflammation or edema and not wallerian degeneration or myelomalacia. It is also important to note that tract density elevation at the site of compression on DTI reflects a localized change in the number of tracts per unit area and is actually dependent on both the total number of fibers passing through the lesion site and the cross-sectional area of the cord at the site of compression. Thus, even if there were a reduction in the total number of tracts passing through the lesion site, as was reported in previous studies, these fiber tracts are forced through a smaller cross-sectional area and therefore would likely demonstrate an increase in local fiber tract density at the site of compression.

It is also important to clarify the distinction between fiber tracts generated using probabilistic DTI tractography and physical axon tracts, the latter of which is a mathematical construct dependent on the underlying microstructure of the tissue and may not directly reflect changes within the axon structures themselves. Physiologically, an increase in neurological impairment may be the result of axonal degeneration from chronic compression of the cord resulting from tissue ischemia, injury, and neurological impairment. However, the underlying architecture of the spinal cord may still be intact during compression, resulting in a focal increase in fiber tract density as DTI fibers converge at the site of compression. This may also explain our observation of an increased fiber tract density with increasing neurological impairment. Future studies aimed at correlating the underlying biology with TDI measurements are warranted to fully understand the limitations of this technique in the context of spinal cord evaluation.

While results from the current study are promising, one limitation is that the study does not provide guidance as to how patients with increased tract density respond following surgical intervention. This is beyond the scope of our investigation, yet future prospective studies focused on examining whether TDI can be used to identify patients who respond favorably to therapy are necessary.

\section{Conclusions}

Results from the current study support the use of superresolution TDI as a clinical tool for correlating the area of compression with neurological impairment in patients with cervical spondylosis and myelopathy.

\section{References}

1. Al-Mefty O, Harkey LH, Middleton TH, Smith RR, Fox JL: Myelopathic cervical spondylotic lesions demonstrated by magnetic resonance imaging. J Neurosurg 68:217-222, 1988

2. Behrens TE, Johansen-Berg H, Woolrich MW, Smith SM, Wheeler-Kingshott CA, Boulby PA, et al: Non-invasive mapping of connections between human thalamus and cortex using diffusion imaging. Nat Neurosci 6:750-757, 2003

3. Brain WR, Northfield D, Wilkinson M: The neurological manifestations of cervical spondylosis. Brain 75:187-225, 1952

4. Budzik JF, Balbi V, Le Thuc V, Duhamel A, Assaker R, Cotten A: Diffusion tensor imaging and fibre tracking in cervical spondylotic myelopathy. Eur Radiol 21:426-433, 2011

5. Calamante F, Tournier JD, Heidemann RM, Anwander A, Jackson GD, Connelly A: Track density imaging (TDI): validation of super resolution property. Neuroimage 56:12591266, 2011

6. Calamante F, Tournier JD, Jackson GD, Connelly A: Trackdensity imaging (TDI): super-resolution white matter imaging using whole-brain track-density mapping. Neuroimage 53:1233-1243, 2010

7. Calamante F, Tournier JD, Smith RE, Connelly A: A generalised framework for super-resolution track-weighted imaging. Neuroimage 59:2494-2503, 2012

8. Chang Y, Jung TD, Yoo DS, Hyun JK: Diffusion tensor imaging and fiber tractography of patients with cervical spinal cord injury. J Neurotrauma 27:2033-2040, 2010

9. Demir A, Ries M, Moonen CT, Vital JM, Dehais J, Arne P, et al: Diffusion-weighted MR imaging with apparent diffusion coefficient and apparent diffusion tensor maps in cervical spondylotic myelopathy. Radiology 229:37-43, 2003

10. Descoteaux M, Deriche R, Knösche TR, Anwander A: Deterministic and probabilistic tractography based on complex fibre orientation distributions. IEEE Trans Med Imaging 28:269-286, 2009

11. Ellingson BM, Schmit BD, Kurpad SN: Lesion growth and degeneration patterns measured using diffusion tensor 9.4-T magnetic resonance imaging in rat spinal cord injury. J Neurosurg Spine 13:181-192, 2010

12. Facon D, Ozanne A, Fillard P, Lepeintre JF, Tournoux-Facon $C$, Ducreux D: MR diffusion tensor imaging and fiber tracking in spinal cord compression. AJNR Am J Neuroradiol 26:1587-1594, 2005

13. Fernández de Rota JJ, Meschian S, Fernández de Rota A, Urbano V, Baron M: Cervical spondylotic myelopathy due to chronic compression: the role of signal intensity changes in magnetic resonance images. J Neurosurg Spine 6:17-22, 2007

14. Hori M, Okubo T, Aoki S, Kumagai H, Araki T: Line scan diffusion tensor MRI at low magnetic field strength: feasibility study of cervical spondylotic myelopathy in an early clinical stage. J Magn Reson Imaging 23:183-188, 2006

15. Jones JG, Cen SY, Lebel RM, Hsieh PC, Law M: Diffusion tensor imaging correlates with the clinical assessment of disease severity in cervical spondylotic myelopathy and predicts outcome following surgery. AJNR Am J Neuroradiol 34:471-478, 2013

16. Kara B, Celik A, Karadereler S, Ulusoy L, Ganiyusufoglu K, Onat L, et al: The role of DTI in early detection of cervical spondylotic myelopathy: a preliminary study with 3-T MRI. Neuroradiology 53:609-616, 2011

17. Kerkovský M, Bednarík J, Dušek L, Sprláková-Puková A, Urbánek I, Mechl M, et al: Magnetic resonance diffusion tensor imaging in patients with cervical spondylotic spinal cord compression: correlations between clinical and electrophysiological findings. Spine (Phila Pa 1976) 37:48-56, 2012

18. Lee JW, Kim JH, Park JB, Park KW, Yeom JS, Lee GY, et al: Diffusion tensor imaging and fiber tractography in cervical compressive myelopathy: preliminary results. Skeletal Radiol 40:1543-1551, 2011

19. Mamata H, Jolesz FA, Maier SE: Apparent diffusion coefficient and fractional anisotropy in spinal cord: age and cervical spondylosis-related changes. J Magn Reson Imaging 22:38-43, 2005

20. Mastronardi L, Elsawaf A, Roperto R, Bozzao A, Caroli M, Ferrante M, et al: Prognostic relevance of the postoperative evolution of intramedullary spinal cord changes in signal in- 
tensity on magnetic resonance imaging after anterior decompression for cervical spondylotic myelopathy. J Neurosurg Spine 7:615-622, 2007

21. Matsuda Y, Miyazaki K, Tada K, Yasuda A, Nakayama T, Murakami H, et al: Increased MR signal intensity due to cervical myelopathy. Analysis of 29 surgical cases. J Neurosurg 74:887-892, 1991

22. Matsumoto M, Toyama Y, Ishikawa M, Chiba K, Suzuki N, Fujimura Y: Increased signal intensity of the spinal cord on magnetic resonance images in cervical compressive myelopathy. Does it predict the outcome of conservative treatment? Spine (Phila Pa 1976) 25:677-682, 2000

23. Mehalic TF, Pezzuti RT, Applebaum BI: Magnetic resonance imaging and cervical spondylotic myelopathy. Neurosurgery 26:217-227, 1990

24. Mizuno J, Nakagawa H, Chang HS, Hashizume Y: Postmortem study of the spinal cord showing snake-eyes appearance due to damage by ossification of the posterior longitudinal ligament and kyphotic deformity. Spinal Cord 43:503-507, 2005

25. Mizuno J, Nakagawa H, Inoue T, Hashizume Y: Clinicopathological study of "snake-eye appearance" in compressive myelopathy of the cervical spinal cord. J Neurosurg 99 (2 Suppl):162-168, 2003

26. Morio Y, Yamamoto K, Kuranobu K, Murata M, Tuda K: Does increased signal intensity of the spinal cord on MR images due to cervical myelopathy predict prognosis? Arch Orthop Trauma Surg 113:254-259, 1994

27. Nakamura M, Fujiyoshi K, Tsuji O, Konomi T, Hosogane N, Watanabe K, et al: Clinical significance of diffusion tensor tractography as a predictor of functional recovery after laminoplasty in patients with cervical compressive myelopathy. J Neurosurg Spine 17:147-152, 2012

28. Parker GJ, Alexander DC: Probabilistic anatomical connectivity derived from the microscopic persistent angular structure of cerebral tissue. Philos Trans R Soc Lond B Biol Sci 360:893-902, 2005

29. Pavlov H, Torg JS, Robie B, Jahre C: Cervical spinal stenosis: determination with vertebral body ratio method. Radiology 164:771-775, 1987

30. Puzzilli F, Mastronardi L, Ruggeri A, Lunardi P: Intramedullary increased MR signal intensity and its relation to clinical features in cervical myelopathy. J Neurosurg Sci 43:135139,1999

31. Ramanauskas WL, Wilner HI, Metes JJ, Lazo A, Kelly JK: MR imaging of compressive myelomalacia. J Comput Assist Tomogr 13:399-404, 1989

32. Sato T, Horikoshi T, Watanabe A, Uchida M, Ishigame K, Araki T, et al: Evaluation of cervical myelopathy using apparent diffusion coefficient measured by diffusion-weighted imaging. AJNR Am J Neuroradiol 33:388-392, 2012

33. Schwartz ED, Cooper ET, Fan Y, Jawad AF, Chin CL, Nissanov J, et al: MRI diffusion coefficients in spinal cord correlate with axon morphometry. Neuroreport 16:73-76, 2005
34. Suk KS, Kim KT, Lee JH, Lee SH, Kim JS, Kim JY: Reevaluation of the Pavlov ratio in patients with cervical myelopathy. Clin Orthop Surg 1:6-10, 2009

35. Tournier JD, Calamante F, Connelly A: Robust determination of the fibre orientation distribution in diffusion MRI: nonnegativity constrained super-resolved spherical deconvolution. Neuroimage 35:1459-1472, 2007

36. Uda T, Takami T, Tsuyuguchi N, Sakamoto S, Yamagata T, Ikeda $\mathrm{H}$, et al: Assessment of cervical spondylotic myelopathy using diffusion tensor magnetic resonance imaging parameter at 3.0 tesla. Spine (Phila Pa 1976) 38:407-414, 2013

37. Wada E, Ohmura M, Yonenobu K: Intramedullary changes of the spinal cord in cervical spondylotic myelopathy. Spine (Phila Pa 1976) 20:2226-2232, 1995

38. Wen CY, Cui JL, Lee MP, Mak KC, Luk KD, Hu Y: Quantitative analysis of fiber tractography in cervical spondylotic myelopathy. Spine J 13:697-705, 2013

39. Xiangshui M, Xiangjun C, Xiaoming Z, Qingshi Z, Yi C, Chuanqiang Q, et al: 3 T magnetic resonance diffusion tensor imaging and fibre tracking in cervical myelopathy. Clin Radiol 65:465-473, 2010

40. Yonenobu K, Abumi K, Nagata K, Taketomi E, Ueyama K: Interobserver and intraobserver reliability of the Japanese Orthopaedic Association scoring system for evaluation of cervical compression myelopathy. Spine (Phila Pa 1976) 26:1890-1895, 2001

41. Young WF: Cervical spondylotic myelopathy: a common cause of spinal cord dysfunction in older persons. Am Fam Physician 62:1064-1070, 2000 (Erratum in Am Fam Physician 63:1916, 2001)

42. Yue WM, Tan SB, Tan MH, Koh DC, Tan CT: The Torg-Pavlov ratio in cervical spondylotic myelopathy: a comparative study between patients with cervical spondylotic myelopathy and a nonspondylotic, nonmyelopathic population. Spine (Phila Pa 1976) 26:1760-1764, 2001

\section{Author Contributions}

Conception and design: Ellingson, Salamon, Holly. Acquisition of data: Ellingson, Salamon, Holly. Analysis and interpretation of data: all authors. Drafting the article: Ellingson, Holly. Critically revising the article: all authors. Reviewed submitted version of manuscript: all authors. Approved the final version of the manuscript on behalf of all authors: Ellingson. Statistical analysis: Ellingson. Administrative/technical/material support: Ellingson.

\section{Correspondence}

Benjamin M. Ellingson, Department of Radiological Sciences, David Geffen School of Medicine, University of California Los Angeles, 924 Westwood Blvd., Ste. 615, Los Angeles, CA 90024. email: bellingson@mednet.ucla.edu. 